\title{
Nano Fiber Images Thresholding based on Imperial Competitive Algorithm
}

\author{
Neda Dehghan \\ Textile Engineering \\ Department, University of Yazd, \\ Yazd, Iran
}

\author{
Pedram Payvandy \\ Textile Engineering \\ Department, University of Yazd, \\ Yazd, Iran
}

\author{
Mohamad Ali Tavanaie \\ Textile Engineering \\ Department, University of Yazd, \\ Yazd, Iran
}

\begin{abstract}
Nano fibers are widely used in various industries, therefor knowing the morphology is important. Thresholding is a simple but effective technique for image segmentation. The goal of image segmentation is to cluster pixels into salient image regions, i.e., regions corresponding to individual surfaces, objects, or natural parts of objects. In this paper a novel method is proposed for performing image segmentation. The purpose of this paper proposed an imperial competitive algorithm with the objective function from Kmeans clustering algorithm for Nano fibers image thresholding. Then algorithm used, with the algorithms such as: global threshold, local threshold, Kmeans clustering algorithm and FCM methods were compared. Finally, a powerful algorithm for image thresholding is found. The comparisons and experimental results show that purposed algorithm is better than other methods particularly global and local thresholding, Kmeans and even FCM.
\end{abstract}

\section{General Terms}

Heuristic Algorithm, Thresholding Method.

\section{Keywords}

Imperial Competitive Algorithm, Segmention, Thresholding, Kmeans Clustering, Nano Fiber.

\section{INTRODUCTION}

Image segmentation plays an important role in image analysis. Based on properties of pixels such as color or intensity image segmentation an image into different segments. Different kinds of segmentation methods have been developed. According to reference [1] four categories of image segmentation are thresholding, clustering, edge detection and region extraction. Threshold based techniques are mainly used for segmenting images consisting of bright objects against dark backgrounds and vice versa. Threshold is one of the most common image segmentation techniques.

Thresholding is a simple but effective technique for image segmentation. Finding a suitable thresholding value to segment an arbitrary object from its background is an important step in image processing and has wide applications in image processing. The thresholding technique is based on partitioning an image into different regions (that is, object and background) in which one sector consists of pixels more than or equal to the proposed threshold value and the other consists of pixels less than the proposed threshold value [2].

Thresholding techniques can generally be categorized into global and local thresholding. Global thresholding selects a single threshold value from the histogram of the entire image, however, local thresholding method segments the image into small images and chooses different value of threshold for different small regions of image (sub image) [3].
Over the past years, methods of threshold selection have been studied by various researchers. Sahoo et al. [4] considered the Otsu method as an appropriate method to determine the threshold for general real world images. Otsu method [5] is an optimal technique of global thresholding, this technique is effective, simple and suitable to automatically select the threshold, align with maximization of between class-variance of two data sets of the histogram which is tantamount to the Minimization of within-class variance. Investigations on offering new algorithms based on Otsu' method have been carried out to improve the efficiency of the method for images containing multiple (multimodal) distribution $[2,6,7]$.

Bansal et al. [3], comparing Otsu and iterative method, introduced the Otsu's method appropriate and accurate to select the optimal threshold. Niblack in [8] introduced the local thresholding method superior to the global thresholding in a way that is suitable for poorly and unevenly illuminated images. Niblack proposed local thresholding technique based on local mean and local standard deviation.

Another technique that can be used for image segmentation is a clustering method. Clustering method extracts useful information from data set to identify the behavior of the system. K-means clustering method is the most applicable method for image thresholding. Lin et al. [9] presented $\mathrm{k}$ means algorithm suitable to extract the object from the background in color images. Srinivas et al. [10] also used kmeans clustering for image segmentation.

Fuzzy clustering method (FCM) is another technique that has been investigated for thresholding in successive years. Yong et al. [11] used the fuzzy clustering method to determine the threshold. Also, they considered it very useful for noisy images in comparison to other methods like Otsu's method. Padmavathi et al. [12] used fuzzy clustering method for segmenting and extracting an object from the background. Moreover, improved fuzzy clustering algorithm was used by Mahjoub in [13] for color image segmentation. Sivakumar et al. [14] used fuzzy clustering method to segment the mammogram images and considered suitable for thresholding of medical images.

Sivanand in [15] used Local threshold method and fuzzy clustering method. In the study, improved local threshold method was found suitable to avoid the problem of global threshold with noise and low resolution images. Also, fuzzy clustering method was introduced for medical image segmentation.

In clustering methods, the final solution is dependent on the choice of initial cluster centers. Then, one of the disadvantages of the clustering method is that there is no specific procedure for calculating initial cluster centers which is likely to trap the solutions in optimal local points. 
The latest techniques used for image thresholding is the use of heuristic algorithm. In 2013, Soltanpoor et al. [16] used imperialist competitive algorithm for image segmentation and considered this algorithm as a suitable method for thresholding and object segmentation from the background. The objective function used in this competitive algorithm is concerned with information based on image graph.

Understanding the morphology of the Nano fiber structures to know the fiber size distribution and their pores is necessary for some applications. Therefore, the quick and accurate measurement of these characteristics is required an appropriate method. Image processing as an efficient, reliable and very quick method to measure and extract structural characteristics of Nano fiber images has been used. In this paper, a novel method to thresholding the Nano fiber images based on the optimization of imperialist competitive algorithm in line with the objective function inspired by k-means clustering method has been proposed. Then, the results obtained for the proposed method has been compared to other methods of thresholding such as global thresholding, local thresholding, and thresholding with the use of k-means and FCM algorithms.

\section{PROBLEM STATEMENT}

Nano fibers are those which their diameters are less than 1 micron. When the diameter of polymeric fiber lessens from one micrometer to less than one micrometer or hundreds of nanometer, new and special features including great mechanical properties, flexibility due to the decrease of diameter, increase of the surface to volume of fiber, reduction of pore size are emerged. These striking properties have led Nano fiber structures to be an appropriate choice for many important applications with high efficiency. Figure (1) shows Nano fiber web.

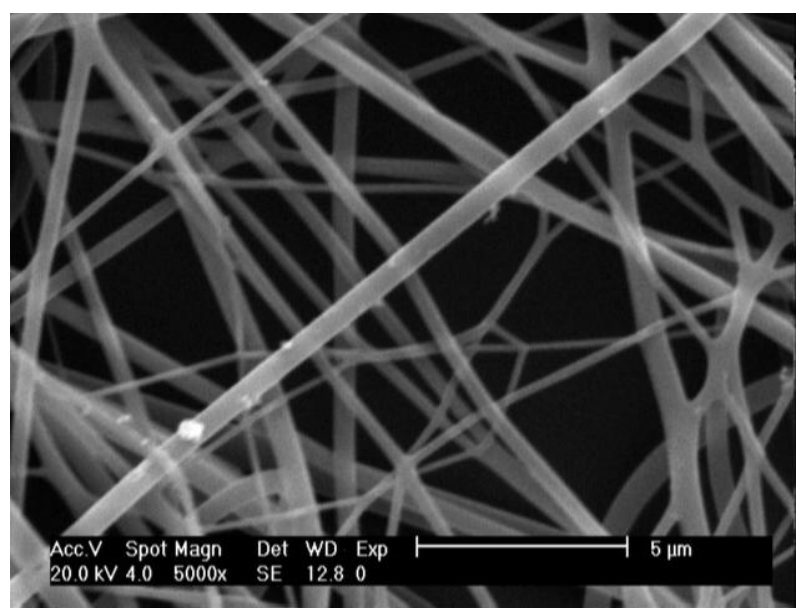

Fig 1: Micrographs of the electrospun Nano fiber webs

Physical and mechanical properties of the Nano fiber webs and nonwoven textile are not only dependent on the properties of the made materials, but also on their structural characteristics. The tiny size of fiber and different fiber orientation has resulted to the difficult measurement of Nano fiber pores and diameter. In recent years, image analyzing method to measure these properties such as: fiber orientation, uniformity, diameter and Nano fiber pore web measurement have been developed.

Pourdeyhimi et al. $[17,18]$ studied orientation in fibers; they also considered necessary the suitable selection of threshold to segment fibers for the background. Fiber uniformity is one of the Nano fiber properties which were investigated by Chhabra et al. [19]. In study by Ziabari et al. [20], direct tracking method to measure the Nano fiber diameter was used. Also, this study applied local thresholding method to threshold Nano fiber images. Local thresholding method for thresholding Nano fiber web images to measure diameter has been used in other investigations [21-23]. In another investigation [24] to measure the diameter, threshold value was selected for Nano fiber images on the base of trial and error method. Pore size is another important property of Nano fiber web which has been used by [25-27], to measure pores with image processing.

\section{THRESHOLDING METHODS}

Thresholding is the process of converting a gray scale image to a binary image using an optimum threshold value. It is a process of partitioning an image into object pixels and background pixels. The pixel is related to the object pixel if the pixel value is greater than a certain threshold value, however, less the certain threshold value is related to the background pixel. The methods used for thresholding are global and local thresholding, fuzzy algorithms such as $\mathrm{K}$ means clustering algorithm and Fuzzy C-means.

\subsection{Global Thresholding}

When pixel intensity distribution of the objects and the background are separate, it means image contains the clear object in the dark background or vice versa, one can use the global thresholding on entire image.

\subsection{Local Thresholding}

Global thresholding techniques will face trouble if image background is not properly bright and clear. Local thresholding is used to compensate background non-uniform illumination by dividing original image into sub images. The image divided into small parts causes illumination of each part is fairly uniformed.

\subsection{Iterative Thresholding Method}

This method is one of the global thresholding techniques which are working on the base of assuming an initial value of threshold. This algorithm is following these steps:

1. Select an initial estimate of $T$ value which can be at the average point of minimum and maximum intensity value of 1 . 2. Using this value $T$, an image is divided into two pixel regions, T1 (with all pixel values $<\mathrm{T}$ ) and $\mathrm{T} 2$ (with all pixel values $>\mathrm{T})$.

3. Compute the average intensity values $\mu_{1}$ and $\mu_{2}$ of regions $\mathrm{T} 1$ and $\mathrm{T} 2$ respectively.

4. Compute a new value of $\mathrm{T}$ as:

$$
T=\left(\mu_{1}+\mu_{2}\right) / 2
$$

5. Repeat the above steps from 2 to 4 till difference between two successive values of $\mathrm{T}$ becomes minimal [3].

\subsection{Fuzzy C-Means Clustering (FCM)}

One of the most important and applicable clustering algorithms is FCM. Pixels in this algorithm are divided into C clusters. Also, the number of $\mathrm{C}$ is predetermined. This method developed by [28]. The objective function for this algorithm is defined as following:

$$
J=\sum_{j=1}^{N} \sum_{i=1}^{C} u_{i j}^{m}\left|x_{j}-v_{i}\right|^{2}
$$

In equation, $\mathrm{X}=\left(\mathrm{x}_{1}, \mathrm{x}_{2}, \ldots, \mathrm{x}_{\mathrm{N}}\right)$, image characteristics and $\mathrm{N}$ is the number of image pixels which are divided into two clusters. $\mathrm{u}_{\mathrm{ij}}$ is the indicative of pixel membership $\mathrm{x}_{\mathrm{j}}$ in the first cluster or the second, $\mathrm{v}_{\mathrm{i}}$ is the center of the first 
or the second cluster, and this mark $\left.\right|^{*} \mid$ is the similarity value(distance) of sample from the cluster center which can use any function, indicative of sample similarity and cluster center. In the above formulae, $\mathrm{m}$ is an actual number greater than 1 which number 2 is chosen for (m) in many cases. Objective function $\mathrm{J}$ will be minimum, when pixels are closer to their cluster centers and have high membership value and low membership value for pixels farther than the centers.

Membership function and cluster centers are calculated in the following equation:

$$
u_{i j}=\frac{1}{\sum_{k=1}^{c}\left(\frac{\left\|x_{j}-v_{i}\right\|}{\left\|x_{j}-v_{k}\right\|}\right) \frac{2}{m-1}}
$$

And

$$
v_{i}=\frac{\sum_{j=1}^{N} u_{i j}^{m} x_{j}}{\sum_{j=1}^{N} u_{i j}^{m}}
$$

Algorithm steps:

1. Initial amount for $\mathrm{C}, \mathrm{m}$ and $\mathrm{u}_{0}$ initial clusters have been assumed.

2. Cluster centers must be calculated $\left(\mathrm{v}_{\mathrm{i}}\right)$

3. Calculate membership matrix.

4. If $\|u l+1-u l\| \leq \varepsilon$, algorithm ends, otherwise return to step 2 .

\section{PROPOSED ALGORITHM}

In this research, ICA is used as an algorithm to threshold the image that the objective function of this algorithm is inspired by k-means clustering. Nano fiber images used for Nano fiber web have made of Electro spinning polyvinyl alcohol with average molecular Weight of $72000 \mathrm{~g} / \mathrm{mol}$. Images are gilded using Scanning Electron Microscope after gold sputter coating. Used images are changed into gray scale image. Preprocessing images like equalizations and denoising have been done on images.

\subsection{Image preprocessing}

\subsubsection{Image Histogram Equalization}

To identify fibers and pores in Nano fiber images, it is required to have an intensity difference between fiber pixels and pores. Image equalization with the increase of intensities of fiber to the image background can make intensity difference for fibers and pores.

\subsubsection{Image Denoising}

One of the effective ways to lessen the noise is to use median linear filter. In this method with the use of defining neighborhood around the related pixel in the input image, output pixel of median value of that neighborhood is around the input pixel. Figure (2) shows a Nano fiber image with an image histogram that the present image is the contrast enhancement, and the image after denoising.

\subsection{K-means clustering method}

Clustering or cluster analysis is a branch of data analysis which assigns the data to a number of predetermined clusters using common data features and no need of the default data. Cluster is a set of objects which are similar, and dissimilar to other objects. Different factors can be considered for similarity, one of which is a distance factor that can be used for clustering. Then, the closer objects regard as one cluster. This clustering is also called clustering based on distance. $\mathrm{K}$ means algorithm is one of the most useful clustering algorithms. $\mathrm{K}$ means algorithm is defined as following:

Step 1: select K data as a center of the cluster.

Step 2: determine the distance of other data to the cluster centers.

Step 3: place the data in the cluster which is closer to any cluster.
Step 4: calculate each mean cluster as a new cluster center. Step 5: repeat steps 2 to 4 to get the stable state in clusters.

Finding number of optimal clusters is usually called cluster validation problem [29]. Therefore, many studies have been carried out by researchers. Among them, one may point to investigations done by [30-32]. Clustering validation index mainly uses two criteria of compression and dispersion to evaluate clustering. These indexes have attempted to calculate compression and separation between clusters, in some cases, their overlap and appropriate production of their components to find the most suitable clustering. These indexes are Dunn index [33], Davies Bouldin Index [34], index validation of the root mean square deviation [35], SD validation index [36] and etc.

\subsection{Imperialist comparative algorithm}

Imperialist comparative algorithm was proposed by AtashPaz and Lukas [37] for the first time. This algorithm has regarded the imperialist process as a stage to the social-political evolution. Also, math modeling of this historical phenomenon as a source of inspiration enjoys the powerful algorithm in optimization. Imperialist competitive algorithm has initiated with a number of initial random population of which each is called a country. Numbers of the best population elements are selected as "imperialist". The rest of the population is considered as "colony". Imperialists depending on their power draw these colonies toward themselves. The total power of this empire is dependent on both constituents, that means imperialist country (as a central core) and its colonies. In mathematics, this dependence with the definition of empire power as a total power of the imperialist country, plus colonies' power mean percentage has been modeled.

With the formation of initial empires, imperialist competitive has started among them. Any country which cannot act successfully in imperialist competitive and increase its power will be eliminated from the scene of imperialist competitive. Therefore, the existence of each empire is dependent on its power to draw colonies of rival empires to itself and occupy them. As a result, in the imperialist competitive trend.

Imperialists based on their power, draw these colonies toward them. Therefore, the existence of any empire is dependent on its power to gain other empire colonies and occupy them. As a result, in the imperialist competitive trend, the power of greater empires will be gradually increased and the weaker empires will be eliminated. Empires to increase their power are forced to develop their colonies. By passing time, colonies will become closer to the empires based on the power and there happens kind of convergence. The ultimate limit of imperialist competitive is when there remains a united empire in which there are colonies very close to the imperialist country regarding the position [37]. Details of the ICA approach are illustrated in flowchart of Figure 3.

\subsubsection{Establishing initial countries}

At the beginning of implementing this algorithm, countries must be made randomly. In optimization algorithm, the aim is to find the optimal solution in terms of variables of the problem. In this step, an array of problem variables is made to be optimized. In a solving a $N_{V a r}$ dimensional optimization problem, a country is $1 \times N_{V a r}$ array. This array is defined as such:

As the first step, some arbitrary initial population is selected. An array of optimization variables is then constructed. When solving a $N_{V a r}$ dimensional optimization problem, a country is $1 \times N_{V a r}$ array. This array is defined as such:

$$
\text { country }=\left[p_{1}, p_{2}, p_{3}, \ldots, p_{i}, \ldots, p_{N_{v a r}}\right]
$$

That $P_{i}=\left(1, \ldots, N_{V a r}\right)$ denotes the optimization variables. 


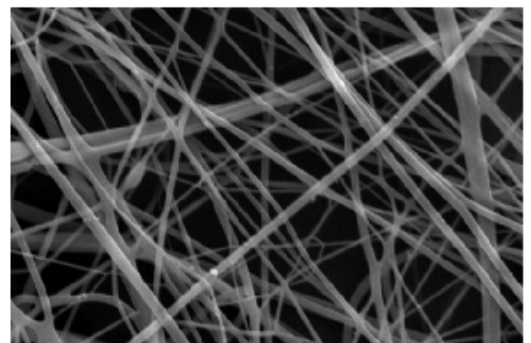

(a)

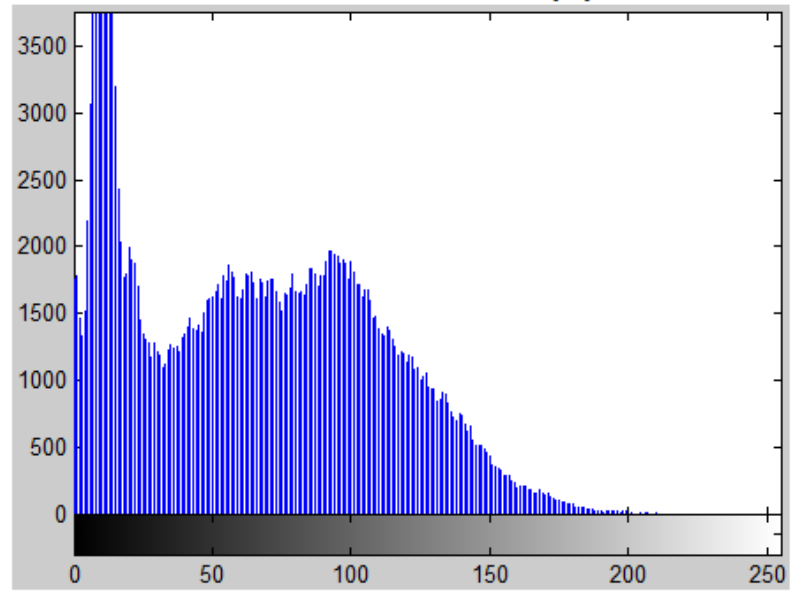

(d)

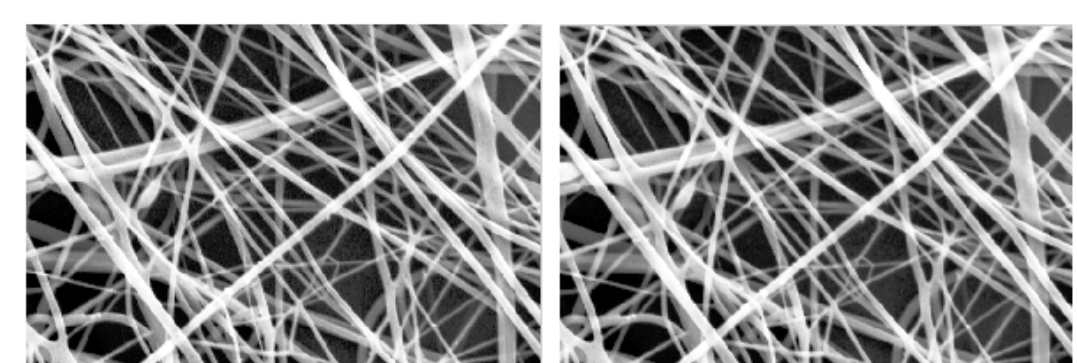

(c)

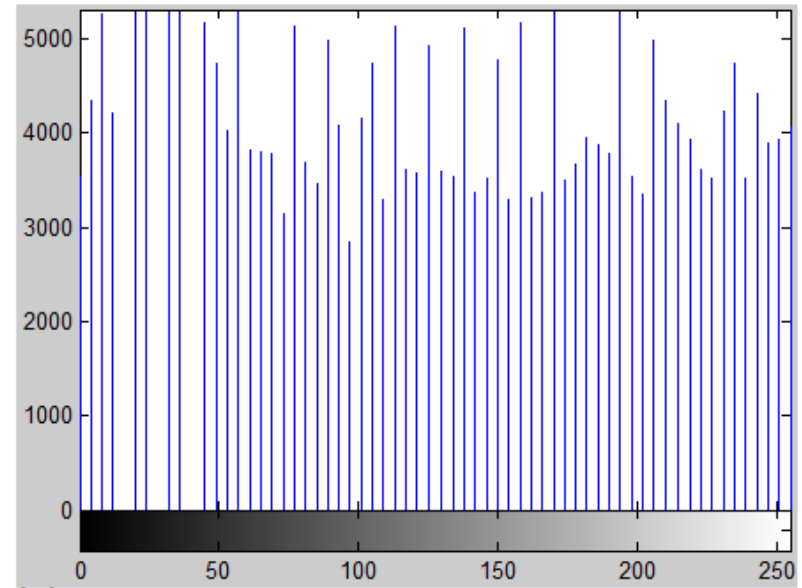

(e)

Fig 2: SEM micrograph of a polymer nanofiber membrane sample: (a) original image; (b) the same image after equalization, (c) image after denoising, (d) Histogram of the original image, (e) ) Histogram of image after equalization

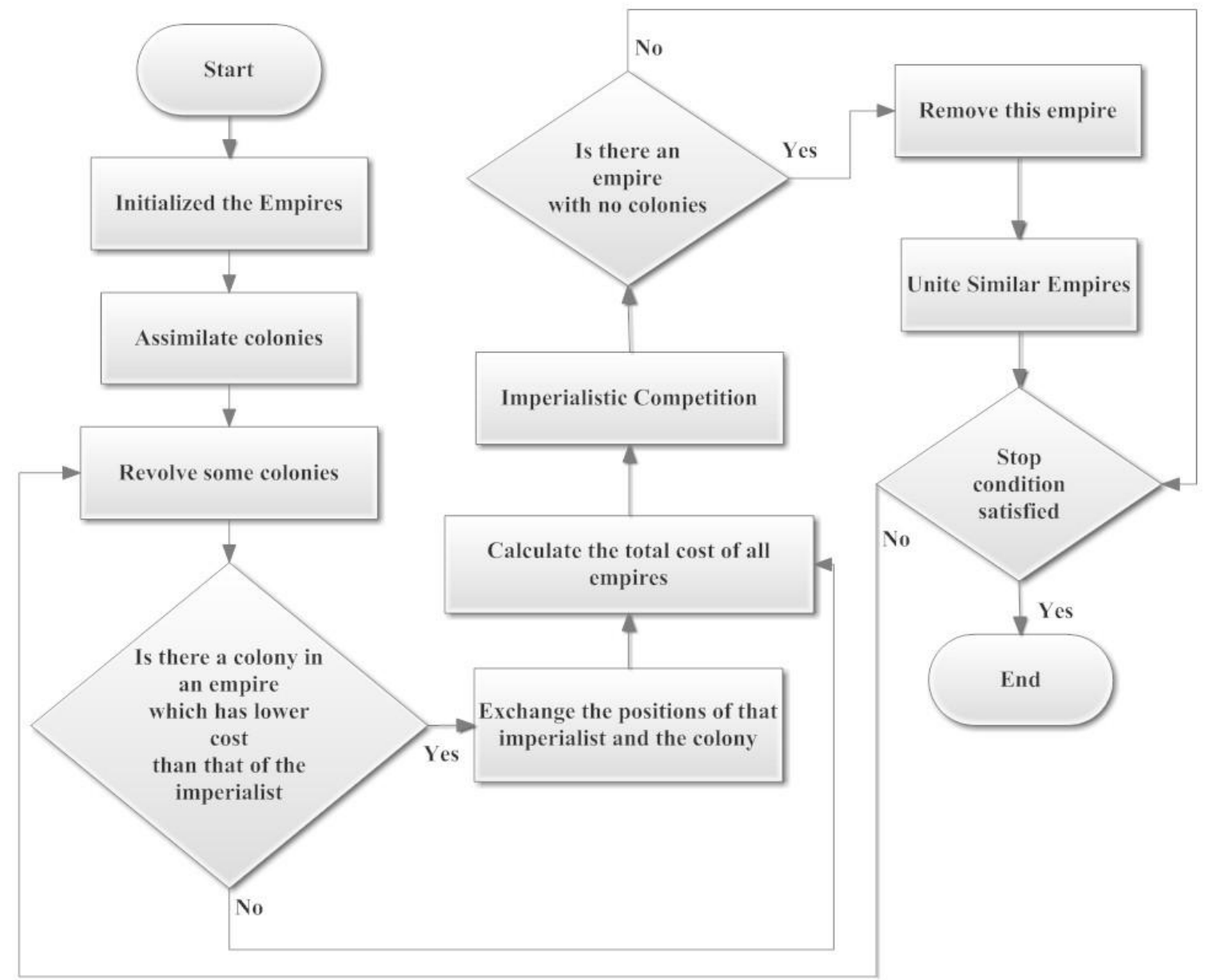

Fig 3: The ICA flowchart 
The optimization terminates after finding the solution with minimum cost value. Initial countries of size $\mathbf{N}_{\text {Country }}$ are produced in the next step. Then, $\mathbf{N}_{\mathbf{i m p}}$ numbers of strong countries are considered as $\mathbf{N}_{\mathbf{i m p}}$ imperialists. The rest with the size $\mathbf{N}_{\text {Col }}$ will form the colonies that belong to an empire. Then, the colonies are assigned to imperialists according to their power. The formations of initial empires are shown in Figure (4). Regarding Figure (4), more powerful imperialists have more colonies.

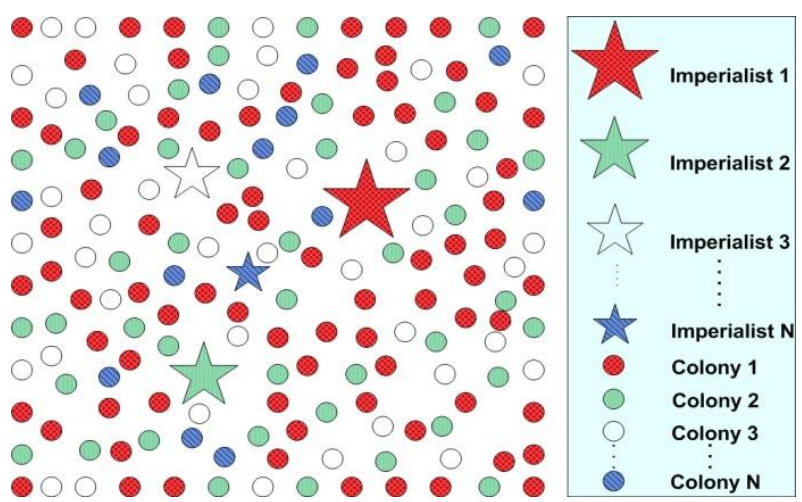

Fig 4: Initialize the empires and their colonies

\subsubsection{Objective function}

In objective function used for ICA algorithm like k-means clustering algorithm, data belonging to a cluster is determined and identified. In a way that data are attributed to the closer cluster with the distance measurement to cluster centers. Choosing number of clusters, minimum distance of Euclidean data from the cluster centers is obtained by using this equation which is a criterion for the cluster compression:

$$
d_{i}=\min _{j}\left(\left\|x_{i}-c_{j}\right\|\right)
$$

In this equation $x_{i}=\left(x_{1}, x_{2}, \ldots, x_{n}\right)$, Image data include pixel intensity of image and $n$ is the number of input data. $c_{j}=$ $\left(c_{1}, c_{2}, \ldots, c_{k}\right)$ cluster centers which are made in the process of imperialist competitive among countries and $\mathrm{k}$ is the number of cluster ( $\mathrm{K}=3$ in threshold problem) .cluster separation criterion which is calculated by distance calculation between cluster centers from each other and is used in objective function.

$$
D=\left(\left\|c_{i}-c_{j}\right\|\right)
$$

$\mathrm{C}_{\mathrm{i}}$ is cluster center (i). In objective function, two criteria of minimum sum of data distances from the center and sum of cluster distances are used. Function objective is defined as such:

$$
\text { Objective }=\sum_{i=1}^{n} \min _{j}\left(\left\|x_{i}-c_{j}\right\|\right)+1 /\left(\sum\left(\left\|c_{i}-c_{j}\right\|\right)+\varepsilon\right)
$$

In this equation, $\varepsilon$ value, minimal value, is used to prevent zero denominators.

\subsubsection{Assimilation}

Imperialists attempt to get colonies close by using assimilation in various socio-political dimensions. Optimization algorithm has modeled this process as colony movement toward imperialist country. Figure 5.a illustrates its overview. As shown in the figure, colony has moved $\mathrm{X}$ unit to the imperialist and has drawn to the new position of colony. In this figure, $\mathrm{d}$ shows the distance between imperialist and colony. $\mathrm{X}$ is a random number with the consistent distribution (or any suitable distribution). It shows $\mathrm{X}$ distribution as below:

$$
X \sim U(0, \beta \times d)
$$

Which $\beta$ is a greater coefficient than 1 and $d$ is a distance between colony and imperialist. The existence of $\beta>1$ makes the colonies close to the imperialist while moving toward the imperialist country in different directions.

According to assimilation algorithm, the imperialist states tend to draw their colonies toward themselves and make them a part of themselves. The assimilation method in the ICA is illustrated by giving a move to all the colonies toward the imperialists. Figure 5(a) illustrates such a movement in which a colony moves toward the imperialist by $x$ which is a random variable with uniform (or any proper) distribution. Where $\beta$ is a coefficient greater than 1 and $d$ is the distance between the colony and the imperialist. If $\beta>1$ then colonies will get closer to the imperialist.

Figure 5 (a) direct movement of colony toward the related imperialist, direct movement of colony toward the imperialist with angle deviation $\mathrm{b}$ in assimilation policy; colony movement toward the imperialist is not necessarily direct. Actually, colony can get close to the imperialist indirectly with the deviation. This probable deviation is done adding a random angle to the imperialist assimilation direction.

Figure 5(b) shows this status. Therefore, instead of moving $X$ to the imperialist country and in the direction of colony to the imperialist, move the same extent, but with $\theta$ deviation in the direction. $\theta$ Movement can be used randomly with the consistent distribution (or any other suitable distribution). Then:

$$
\theta \sim(-\gamma, \gamma)
$$

In this equation $\gamma$ is an optional parameter which its increase makes the increase of exploration around imperialist and its decrease make original colony move close to the imperialist. Assimilating the colonies by the imperialist states did not result in direct movement of the colonies toward the imperialist. That is, the direction of movement is not necessarily the vector from colony to the imperialist. To model this fact and to increase the ability of searching more area around the imperialist, a random amount of deviation is added to the direction of movement. Figure 5(b) shows the new direction. In this figure $\theta$ is a parameter with uniform (or any proper) distribution as;

Where $\gamma$ is a parameter that adjusts the deviation from the original direction. Nevertheless the values of $\beta$ and $\gamma$ are arbitrary.

\subsubsection{Revolution}

Revolution is a critical operation in algorithm that means random change of a colony to new random position. Revolution in algorithm prevents solution trapping in local optimum and provides more exploration space.

\subsubsection{Colony position change with imperialist}

While colony is moving toward the imperialist, it may happen that some colonies gain better positions than imperialist lower cost than the cost of imperialist). In this way, colony and imperialist position is changed and the algorithm continues with the new imperialist position. During the algorithm process, if a colony catches a smaller cost than the imperialist, they will swap their position. 


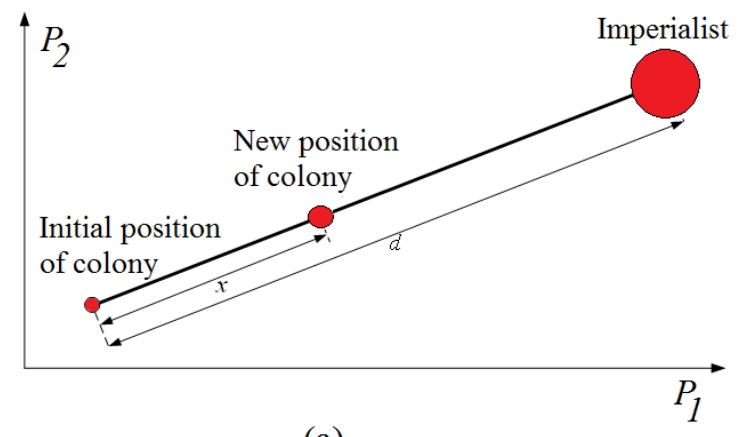

(a)

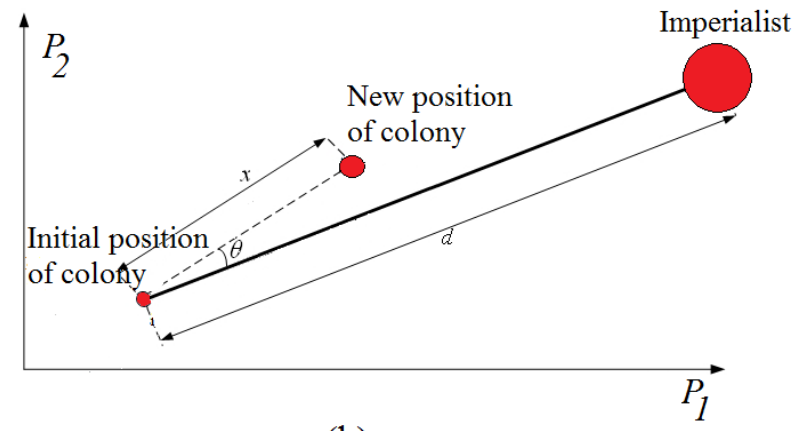

(b)

Fig 5: Direct movement of colonies toward their relevant imperialist (a), Movement of colonies toward their relevant imperialist in a randomly deviated direction (b)

\subsubsection{The total power of an empire}

The power of an empire equals to the power of the colonist, plus some percentages of total power of its colonies. It is calculated as followings:

$$
\begin{gathered}
T_{n}=\operatorname{cost}\left(n^{\text {th }} \text { imprialist }\right)+ \\
\left(\xi \times \operatorname{mean}\left\{\operatorname{cost}\left(\text { colonies of } n^{\text {th }} \text { empire }\right)\right\}\right)
\end{gathered}
$$

Where $T_{n}$ is the total cost of the nth empire and $\varepsilon$ is a positive value. A small value for $\varepsilon$ results the total power of the empire to be defined by just the imperialist and bigger value will increase the colonies effects in determining the total power of an empire.

\subsubsection{Imperialist competitive}

Any empire which cannot increase its power will be eliminated in the imperialist competitive. In a way, that one or some weak colonies are eliminated from the weakest empires and other empires will occupy as well. The mentioned colonies are not necessarily occupied by the stronger empire, however, the stronger empires are most probable to occupy. Therefore, to occupy weaker colonies, there would be competition among all the empires. Figure 6 shows the mentioned competition strategy. Imperialist competitive parameters are presents in Table (1).

\section{Table 1: The ICA parameters}

\begin{tabular}{l|c}
\hline Parameter & Value \\
\hline Number of imperials & 50 \\
Number of countries & 1000 \\
Assimilation coefficient & 2 \\
Deviation coefficient & 0.1 \\
Colonies share coefficient & 0.5 \\
Revolution rate & 0.05 \\
\hline
\end{tabular}

\section{IMAGE QUALITY EVALUTION}

Identifying and determining the quality of images are the key points in evaluating many important methods in image processing like compression, denoising, and the like. Nowadays, identifying the image quality plays a main role in various applications of image processing. The most precise criterion to determine the image quality is to ask individuals who are regarded as mental methods to identify the image quality. Another kind of identifying image quality is called visual methods. In these methods, a set of math equations is

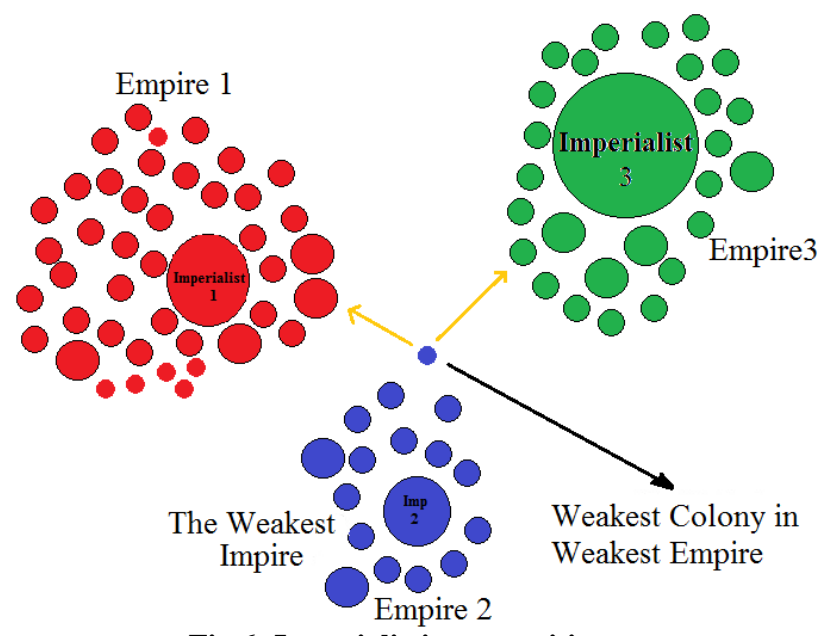

Fig 6: Imperialistic competition

used to assess the error value of the image to the ideal reference image. Since the mental methods are costly and time consuming, one must use methods that consider the sight system of human beings. These methods are called systems based on the human sight system. New methods with the engagement of effective methods in image structure are effective in pixel value change. One of the new factors that image structural characteristics are being used is SSIM4.

This criterion of SSIM is measured on different frames of picture. Measurement between two frames $x$ and $\mathrm{y}$ in $\mathrm{N} \times \mathrm{N}$ size is as follows:

$$
\operatorname{SSIM}(x, y)=\frac{\left(2 \mu_{x} \mu_{y}+c_{1}\right)\left(2 \sigma_{x y}+c_{2}\right)}{\left(\mu_{x}^{2}+\mu_{y}^{2}+c_{1}\right)\left(\sigma_{x}^{2}+\sigma_{y}^{2}+c_{2}\right)}
$$

In equation, $\mu x, \mu y$ stand for means of $\mathrm{X}$ and $\mathrm{Y}$ respectively; also $\sigma x$, are their variances. The constants $C 1, C 2$ are calculated as follows:

$$
\begin{aligned}
& C_{1}=\left(K_{1} L\right)^{2} \\
& C_{2}=\left(K_{2} L\right)^{2}
\end{aligned}
$$

In equations constants $\mathrm{K}_{1}=0.01$ and $\mathrm{K}_{2}=0.03$ and $\mathrm{L}=$ $2^{\neq \text {bits per pixel }-1}$

Quality evaluation of the whole image equals to: 


$$
\operatorname{MSSIM}(X, Y)=\frac{1}{M} \sum_{j=1}^{M} \operatorname{SSIM}\left(x_{j}, y_{j}\right)
$$

In equation, $X$ is the basic image (original) and $Y$ is the output image of each algorithm to be compared. $x j, y j$ are the contents of the $j$ th local view and $M$ is the number of local views in image [38].

In the investigation done, the SSIM index is used to evaluate the quality of the obtained image of the algorithms applied in equation to the reference image as the best image which was determined by asking several experts.

\section{RESULT AND DISCUSSION}

In this study to threshold the Nano fiber images, a new method of imperialist competitive algorithm is used. This method align with other methods such as global, local thresholding methods, and fuzzy methods like Fuzzy C mean (FCM) and K-means clustering algorithm are applied and being compared.

To compare the methods, four images relating to nanofiber were used. Preprocessing of equalization and denoising were done on the images, then the images with the use of global thresholding, local thresholding methods, k means clustering, FCM method and imperialist competitive algorithm became thresholding. In imperialist competitive algorithm, a number of repetitions to get the minimum value of error have been done. Figure (7) shows the change curve and mean curve of cost value change in terms of number of repetition. The results of thresholding are illustrated in Figures 9 to 12.

Main goal of image segmentation is to divide an image into parts that have a strong correlation with objects or areas in the image. In image processing nanofiber pixels in the image are separated from the pores. The result of image segmentation is a set of segments that collectively cover the entire image. In this study, was selected the number of cluster $(K=3)$ for image segmentation in Kmeans, FCM and proposed algorithm, therefor provides more exploration space for segmented pixels intensity of image.

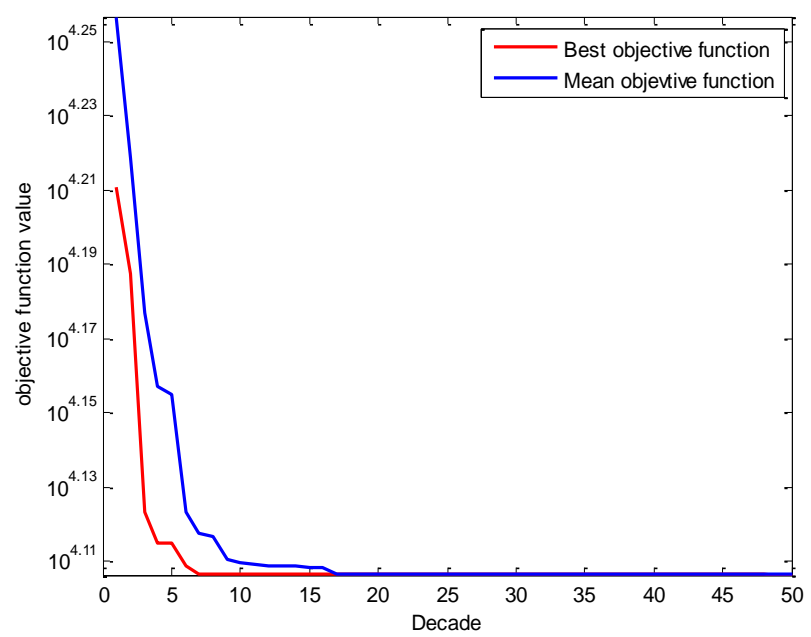

Fig7: Variation of the best and the mean values of the objective function

The image is divided into three clusters, cluster center with the lightest intensity was considered as the first layer. This cluster includes parts of the nanofibers due to their positions are more obvious.

Cluster center of which has the lowest light intensity was considered as the background and was named as the third layer. The remained cluster is located as the second layer in which the cluster center is the middle of the first and the third cluster centers. Figure 8 shows this segmentation.

It seems that the images such as nanofibers ones in which the object has different and combined light intensities, segmentation methods based on the combination of parts to form a two-part image perform better than the usual thresholding method.

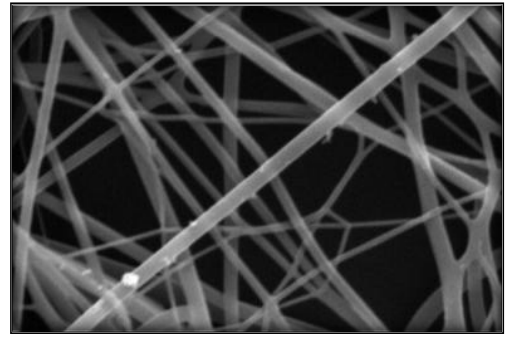

(a)

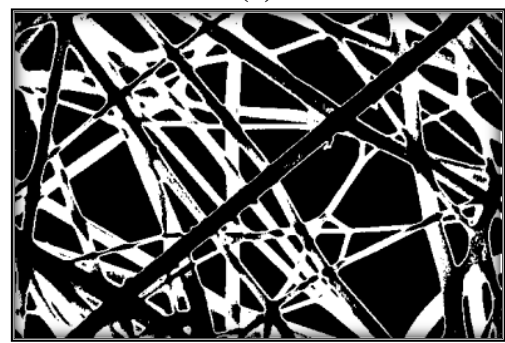

(d)

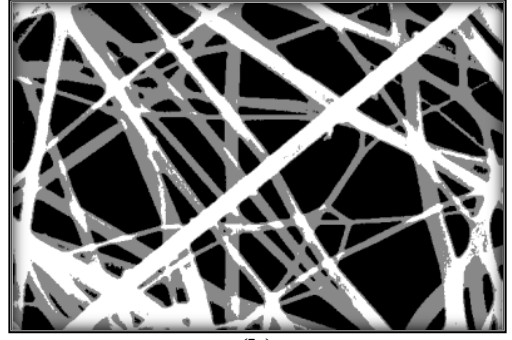

(b)

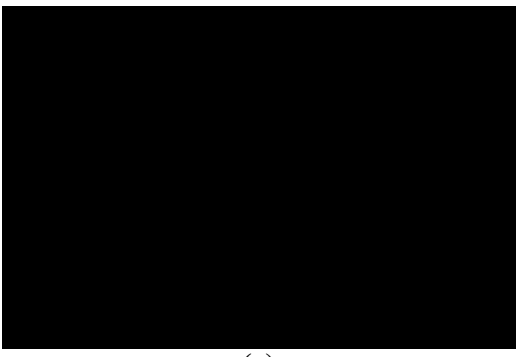

(e)

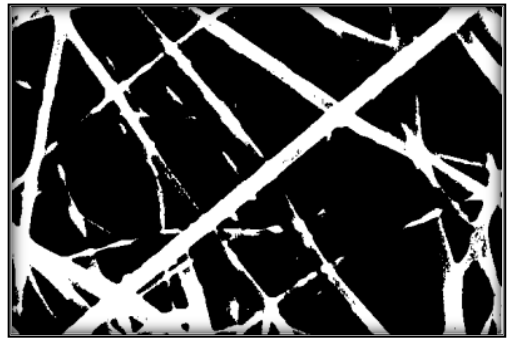

(c)

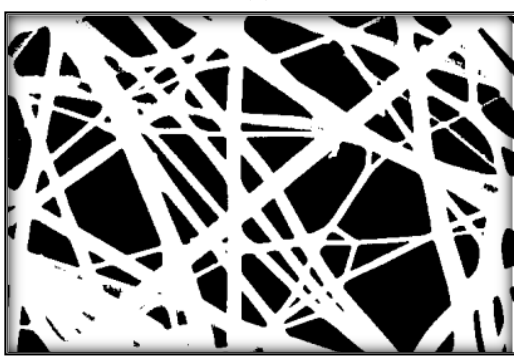

(f)

Fig8: Original image of nanofiber web(a), proposed algorithm segmented image(b), proposed algorithm segmented layer1(c), proposed algorithm segmented layer2(d), proposed algorithm segmented layer3(e),obtained thresholding image(f) 


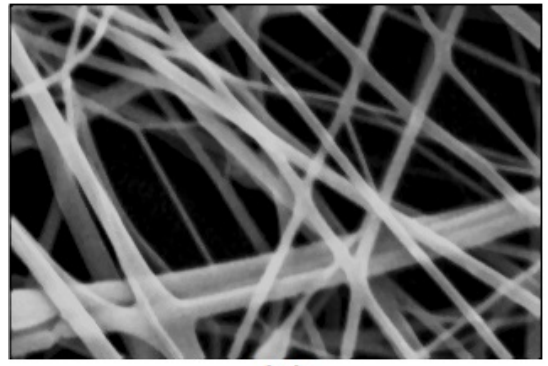

(a)

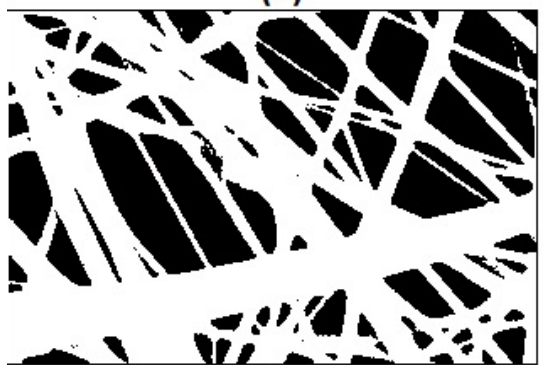

(d)

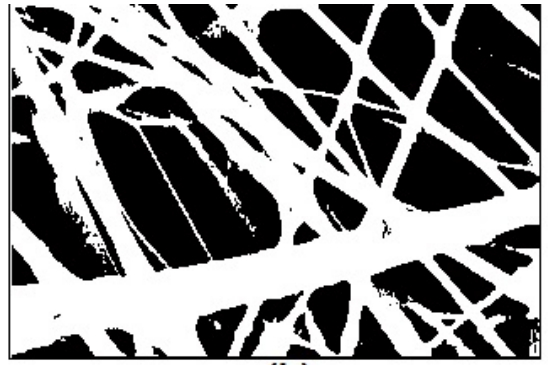

(b)

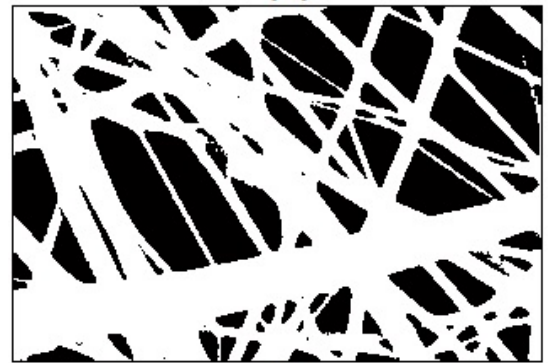

(e)

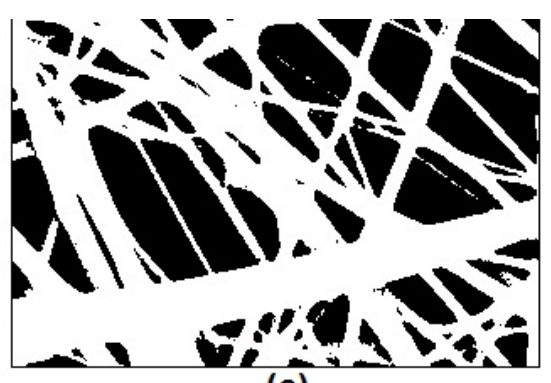

(c)

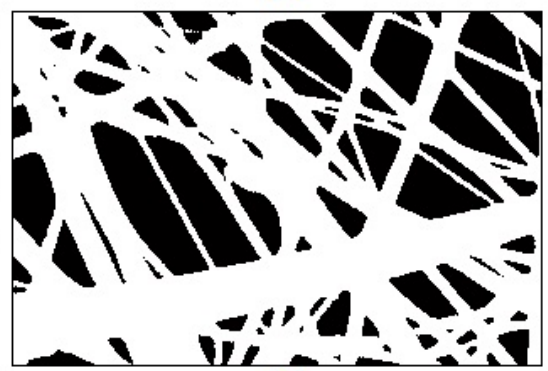

(f)

Fig9: (a) nanofiber image, (b) Otsu segmentation, (c)Local segmentation, (d) Image segmentation by kmeans Clustering , (e) Image segmentation by FCM (f) Image segmentation by imperialist competitive algorithm

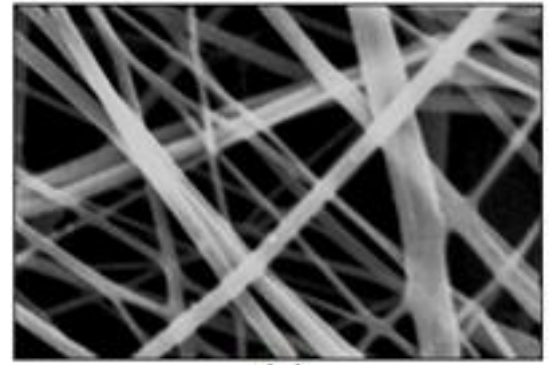

(a)

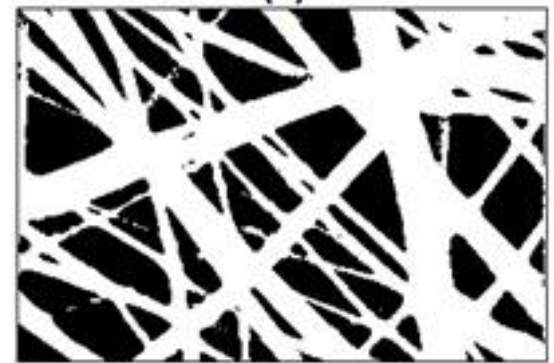

(d)

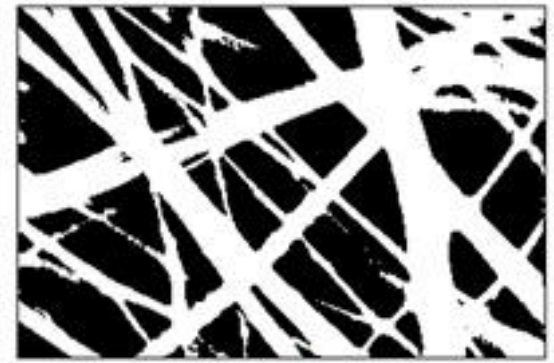

(b)

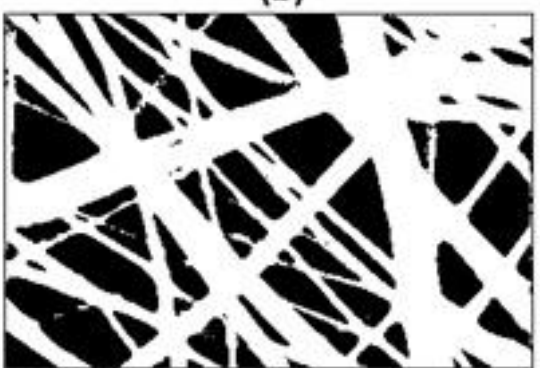

(e)

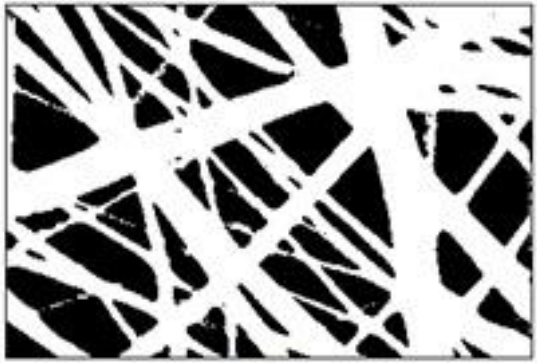

(c)

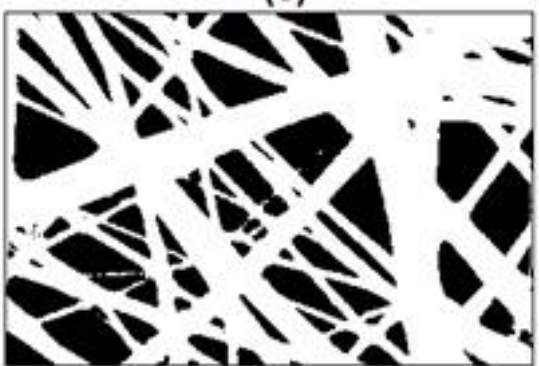

(f)

Fig10: (a) nanofiber image, (b) Otsu segmentation, (c)Local segmentation, (d) Image segmentation by kmeans Clustering , (e) Image segmentation by FCM (f) Image segmentation by imperialist competitive algorithm

In obtained thresholding images with the use of mentioned methods, it has seen that in all figures, the obtained images from local threshold method show better results than global threshold (Otsu). In comparison to FCM and imperialist competitive algorithm methods for nanofiber images, the results of both methods are somehow equal in many cases and in some obtained results from ICA method, it is led to better thresholding. Overall, imperialist competitive algorithm with the objective function inspired by $\mathrm{k}$-means clustering algorithm offer some better results of thresholding. 


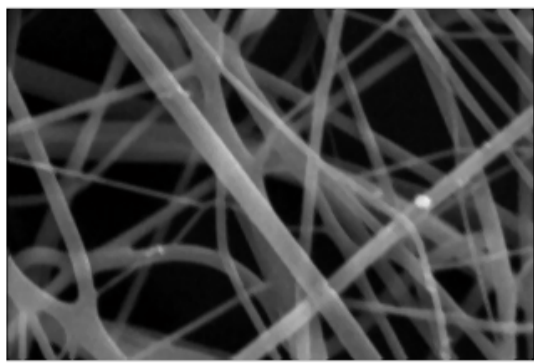

(a)

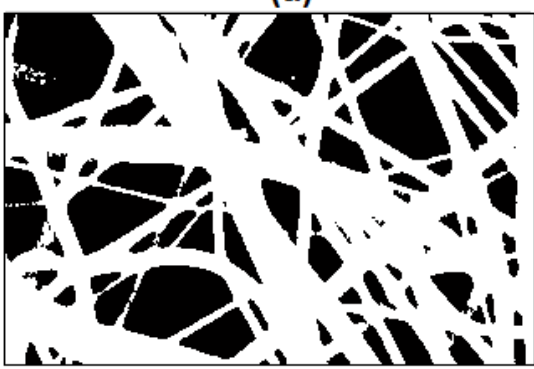

(d)

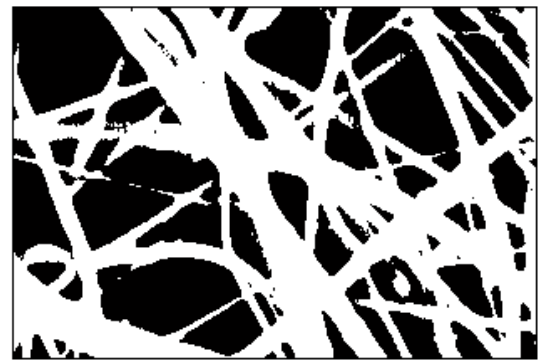

(b)

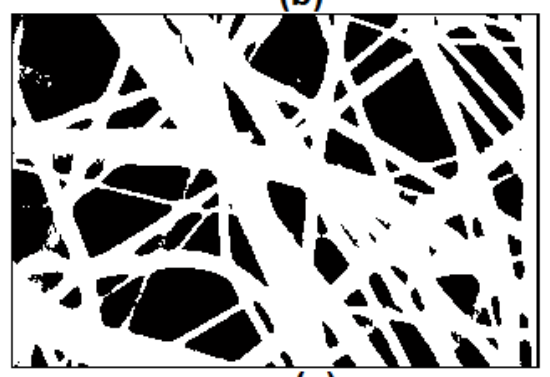

(e)

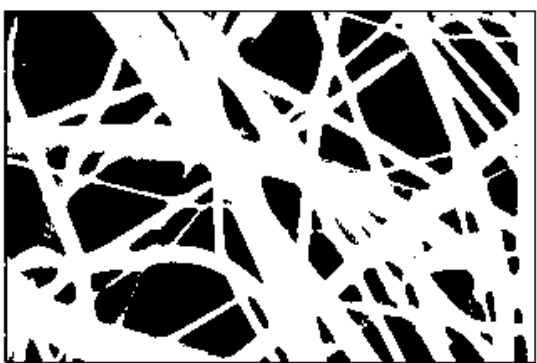

(c)

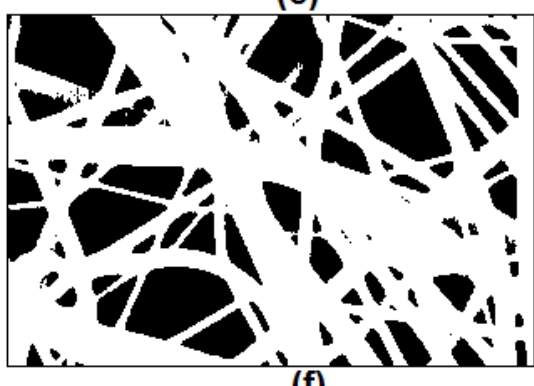

(f)

Fig11: (a) Nano fiber image, (b) Otsu segmentation, (c)Local segmentation, (d) Image segmentation by kmeans Clustering , (e) Image segmentation by FCM (f) Image segmentation by imperialist competitive algorithm

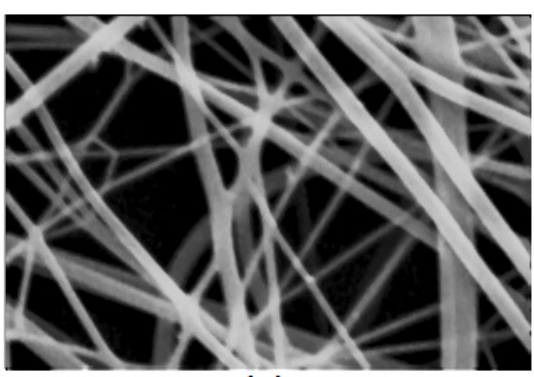

(a)

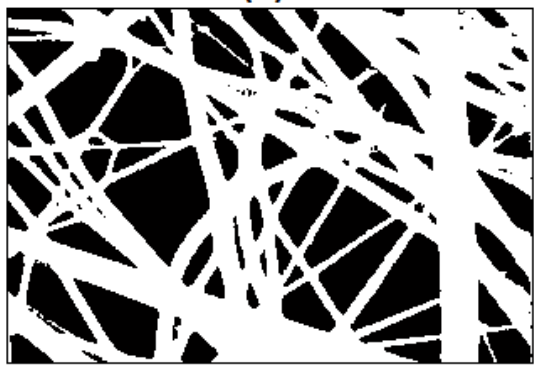

(d)

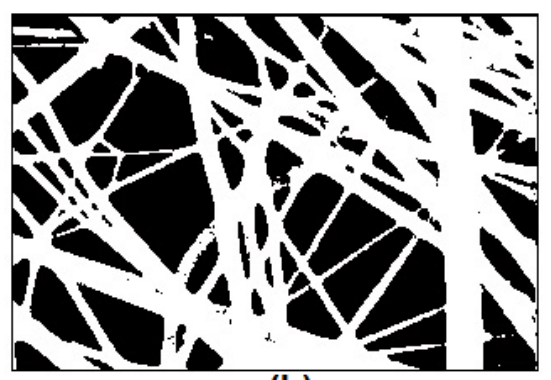

(b)

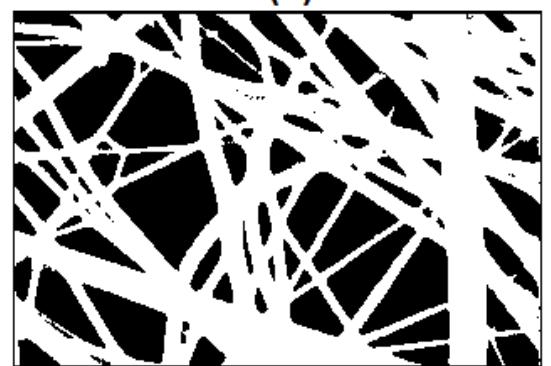

(e)

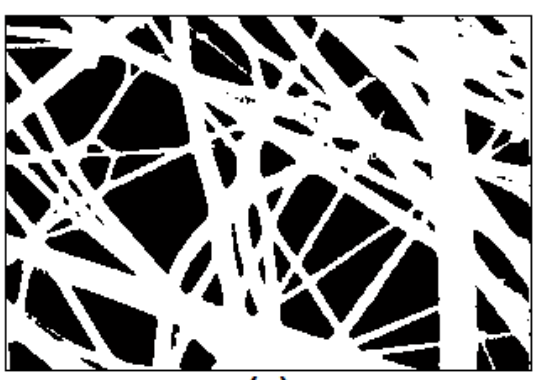

(c)

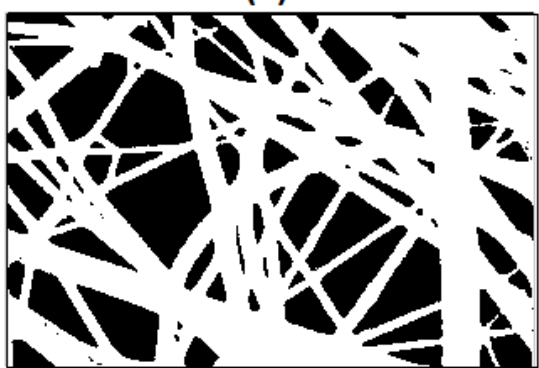

(f)

Fig12: (a) Nano fiber image, (b) Otsu segmentation, (c)Local segmentation, (d) Image segmentation by kmeans Clustering , (e) Image segmentation by FCM (f) Image segmentation by imperialist competitive algorithm

The obtained results from thresholding in Figures (11\&12) similarly show the appropriateness of imperialist competitive algorithm in segmenting the original image from the background.

The obtained quality of thresholding images from the used methods has been evaluated by the use of image quality evaluation method SSIM. Table (2) provides the obtained results from the evaluation of each method and image. The reference image is determined as the best image by asking from some professionals. 
Table 2: quality assessment of different algorithms and comparing to basis method

quality assessment of algorithms quality to
image 9

Whatever the index value of SSIM approximates to1, the obtained image is closer to the reference image. The results of image quality evaluation with the use of SSIM index in Table (2) are like the results from the obtained image of each algorithm in Figures 9 to 12. The outcomes show that local threshold is better than global threshold, and FCM methods and ICA show somehow similar results in the index of SSIM for images. Overall, the index evaluation of SSIM for the gained images from the proposed algorithm is very much closer to 1 which indicates that the imperialist competitive algorithm segments Nano fibers better than the background (pores).

\section{CONCLUSION}

Developing the use of Nano fibers in various industries, knowing the morphology of Nano fiber structures to understand the distribution of fiber size and their pores are to be considered. Time consuming of traditional methods in determining pores, measuring diameters, and also precise measuring of the properties are required to go for quick and accurate methods such as image processing. In this study, this infra heuristic imperialist competitive algorithm with the objective function inspired by k-means clustering algorithm to threshold the Nano fiber images and fiber segmentation have been proposed and accuracy of this method in comparison to global thresholding, local, k-means clustering algorithm and FCM have been studied. In obtained images from thresholding with the proposed algorithm, the number of fibers has been increased and was seen in a more complete way. This means that this method works more successfully in fiber segmentation from pores than other methods. Using the index of image quality evaluation SSIM shows the precision of the applied algorithm. The image and calculating results of thresholding the images of Nano fiber web have justified the precision of using this method than other methods.

\section{REFERENCES}

[1] Fu, B., Mui, S. K., J.K. 1981. A Survey on Image Segmentation. Pattern Recognition, 13, 3-16.

[2] Ng, H. F. 2006. Automatic thresholding for defect detection. Pattern recognition letters, 27(2006), 16441649 .

[3] Bansal, S., and Maini, R. A Comparative Analysis of Iterative and Otsu's Thresholding Techniques. Int. J. Comput. Applications, 66, 45-47.

[4] Sahoo, P. K., Soltani S. A. K. C., and Wong, A. K. (1988). A survey of thresholding techniques. Computer vision, graphics, and image processing, 41, 233-260.

[5] Otsu, N. (1975). A threshold selection method from graylevel histograms. Automatica, 11(285-296), 23-27.

[6] Liao, P. S., Chen, T. S., \& Chung, P. C. (2001). A fast algorithm for multilevel thresholding. J. Inf. Sci. Eng., 17(5), 713-727.

[7] Singh, K. C., Satapathy, L. M., Dash, B., \& Routray, S. K. (2011). Comparative Study on Thresholding. International Journal of Instrumentation Control \& Automation (IJICA), 1(1), 73-77.

[8] Niblack, W., (1986). an Introduction to Digital Image Processing, Prentice-Hall, Englewood Cliffs, NJ.

[9] Lin, W. T., Lin, C. H., Wu, T. H., \& Chan, Y. K. (2010). Image segmentation using the k-means algorithm for texture features. World Academy of Science, Engineering and Technology, 65, 612-615. 
[10] Srinivas, K., and Srikanth, V., (2012). A Scientific Approach for Segmentation and Clustering Technique of Improved K-Means and Neural Networks. Int. J. Adv. Res. Comput. Science Software Eng., 2, 183-189.

[11] Yong, Y., Chongxun, Z., \& Pan, L. (2004). A novel fuzzy c-means clustering algorithm for image thresholding. Measurement Science Review, 4(1), 11-19.

[12] Padmavathi, G., Muthukumar, M., \& Thakur, S. K. (2010). Nonlinear Image segmentation using fuzzy c means clustering method with thresholding for underwater images. Int. J. Comput Science Issues, 7(3), $35-40$.

[13] Mahjoub, M. A. (2011). Improved FCM algorithm applied to color image segmentation. Can. J. Image Processing and Comput Vision, 2(2), 16-19.

[14] Sivakumar, S., \& Chandrasekar, C. (April, 2012). Lungs image segmentation through weighted FCM. In Recent Advances in Computing and Software Systems (RACSS), 2012 International Conference on (109-113). IEEE.

[15] Sivanand, S. (2013). Adaptive Local Threshold Algorithm and Kernel Fuzzy C-Means Clustering Method for Image Segmentation. Proc of IJLTET, Int. J. Latest Trends Eng. Technol, 2(3), 261-265.

[16] Soltanpoor, H., VafaeiJahan, M., \& Jalali, M. (2013). Graph-Based Image Segmentation Using Imperialist Competitive Algorithm. Advances in Computing, 3(2), $11-21$.

[17] Pourdeyhimi, B., Ramanathan, R., \& Dent, R. (1996). Measuring fiber orientation in nonwovens part I: simulation. Text. Res. J., 66(11), 713-722.

[18] Pourdeyhimi, B., \& Dent, R. (1997). Measuring Fiber Orientation in Nonwovens Part IV: Flow Field Analysis. Text. Res. J., 67(3), 181-187.

[19] Chhabra, R. (2003). Nonwoven uniformitymeasurements using image analysis. Int. Nonwoven J., 43-50.

[20] Ziabari, M., Mottaghitalab, V., \& Haghi, A. K. (2009). Application of direct tracking method for measuring electrospun nanofiber diameter. Brazilian Journal of Chemical Engineering, 26(1), 53-62.

[21] Ziabari, M., Mottaghitalab, V., McGovern, S. T., \& Haghi, A. K. (2007). A new image analysis based method for measuring electrospun nanofiber diameter. Nanoscale Res Let, 2(12), 597-600

[22] Kanafchian, M., Valizadeh, M., \& Haghi, A. K. (2011). Prediction of nanofiber diameter for improvements in incorporation of multilayer electrospun nanofibers. Korean J. Chem. Eng., 28(3), 751-755.

[23] Haghi, A. K. (2010). Electrospun nanofiber process control. Cellulose Chem. Technol, 44(9), 343-352.
[24] Shin, E. H., Cho, K. S., Seo, M. H., \& Kim, H. (2008). Determination of electrospun fiber diameter distributions using image analysis processing. Macromol. Res., 16(4), 314-319.

[25] Ziabari, M., Mottaghitalab, V., \& Haghi, A. K. (2008). Evaluation of electrospun nanofiber pore structure parameters. Korean J. Chem. Eng., 25(4), 923-932.

[26] Xu, B. (1996). Measurement of pore characteristics in nonwoven fabrics using image analysis. Cloth Text. Res. J, 14(1), 81-88

[27] Aydilek, A. H., Oguz, S. H., \& Edil, T. B. (2002). Digital image analysis to determine pore opening size distribution of nonwoven geotextiles. J. Comput Civil Eng., 16(4), 280-290.

[28] Bezdek, J.C., Hall, L.O., Clarke, L.P. (1993). Review of MR image segmentation techniques using pattern recognition, Medical Physics, 20(4), 1033-1048.

[29] Bezdek, J. C., (1973) Cluster validity with fuzzy sets, Journal of Cybernetics, 3(3), 58-73.

[30] Fukuyama, Y., \& Sugeno, M. (1989, July). A new method of choosing the number of clusters for the fuzzy c-means method. In Proc. 5th Fuzzy Syst. Symp (Vol. 247).

[31] Xie, X. L., \& Beni, G. (1991). A validity measure for fuzzy clustering. IEEE Trans Pattern Anal Machine Intelligence, 13(8), 841-847.

[32] Kwon, S. H. (1998). Cluster validity index for fuzzy clustering. Electron Lett, 34(22), 2176-2177

[33] Dunn, J. C. (1974). Well-separated clusters and optimal fuzzy partitions. J. Cybern, 4(1), 95-104.

[34] Davies, D.L. and Bouldin, D.W. (1979). A Cluster Separation Measure. IEEE Trans on Pattern Anal and Machine Intelligence, 1(2), 224-227.

[35] Sharma, S.C. (1996). Applied Multivariate Techniques. John Wiley and Sons. Publication, New York, NY, USA (1996).

[36] Halkidi, M., Batistakis, Y., \& Vazirgiannis, M. (2001). On clustering validation techniques. J. of Intelligent Information Systems, 17(2-3), 107-145.

[37] Atashpaz-Gargari, E., Lucas, C., (2007). Imperialist competitive algorithm: an algorithm for optimization inspired by imperialistic competition. IEEE cong. Evol. Comput. Proc. Int. Conf. in Singapore. 4661-4667.

[38] Wang, Z., Bovik, A. C., Sheikh, H. R., \& Simoncelli, E. P. (2004). Image quality assessment: from error visibility to structural similarity. Image Processing, IEEE Transactions on, 13(4), 600-612. 